\title{
Time-Bounded Essential Localization for Wireless Sensor Networks
}

\author{
Wei Cheng, Member, IEEE, ACM, Nan Zhang, Member, IEEE, \\ Xiuzhen Cheng, Senior Member, IEEE, Member, ACM, Min Song, Senior Member, IEEE, Member, ACM, and
} Dechang Chen

\begin{abstract}
In many practical applications of wireless sensor networks, it is crucial to accomplish the localization of sensors within a given time bound. We find that the traditional definition of relative localization is inappropriate for evaluating its actual overhead in localization time. To address this issue, we define a novel problem called essential localization and present the first rigorous study on the essential localizability of a wireless sensor network within a given time bound. Additionally, we propose an efficient distributed algorithm for time-bounded essential localization over a sensor network and evaluate the performance of the algorithm with analysis and extensive simulation studies.
\end{abstract}

Index Terms-Essential localization, relative localization, timebounded localization, wireless sensor networks.

\section{INTRODUCTION}

\section{A. Time-Bounded Localization}

$\mathbf{M}$ ANY military and civil applications of wireless sensor networks require the sensors to be aware of their positions in the physical space [1]-[4]. Such positions can be described as either absolute locations (e.g., a combination of latitude, longitude, and altitude) or relative ones (i.e., the location of a sensor relative to others). The localization problem has been extensively studied in terms of both theoretical analysis on the localizability of a sensor network [5]-[7] and practical techniques for the actual positioning of sensors [8], [9].

Missing from the existing research, however, is the localization of sensors within a given period of time. Such a timebounded localization is extremely important for many practical

Manuscript received October 13, 2011; revised March 16, 2012; accepted May 01, 2012; approved by IEEE/ACM TRANSACTIONS ON NETWORKING Editor G. Xue. Date of publication June 01, 2012; date of current version April 12, 2013. This work was supported in part by the US National Science Foundation under Grants CNS-0644247, CNS-0721669, CNS-0852673, CNS-0963957, CNS-0964060, and CNS-1017662.

W. Cheng is with the Department of Computer Science, University of Massachusetts Lowell, Lowell, MA 01854 USA (e-mail: wcheng@cs.uml.edu).

N. Zhang and X. Cheng are with the Department of Computer Science, The George Washington University, Washington, DC 20052 USA (e-mail: nzhang10@gwu.edu; cheng@gwu.edu).

M. Song is with the Electrical Engineering and Computer Science Department, University of Toledo, Toledo, OH 43606 USA (e-mail: Min.Song@utoledo.edu).

D. Chen is with the Department of Preventive Medicine and Biometrics, Uniformed Services University of the Health Sciences, Bethesda, MD 20814 USA (e-mail: dchen@usuhs.mil).

Color versions of one or more of the figures in this paper are available online at http://ieeexplore.ieee.org.

Digital Object Identifier 10.1109/TNET.2012.2200107 applications. Consider a battlefield scenario as a simple motivating example. The localization of sensors must be accomplished within a short period of time because: 1) it is on the critical time path-i.e., a sensor has to position itself first before annotating the monitored data with geographical information; and 2) the localization process in general requires message exchanges between sensors, making the network more likely to be detected by the enemy. Thus, it is critical for the localization process to complete within a given time bound.

\section{B. Outline of the Technical Results}

We find that the traditional definition of relative localization-i.e., a process that terminates when all sensors obtain their locations in the same coordinate system-is inappropriate for evaluating the actual efficiency of localization in practice. The main reason lies in that part of the localization process can be seamlessly integrated into subsequent payload transmissions without incurring additional communication overhead. For example, we shall demonstrate later in this paper that for a given sensor network topology, localization may require an arbitrarily long period of time according to the traditional definition, but indeed it only needs the time of transmitting a short message before allowing every pair of nodes to have their positions automatically transformed into one coordinate system.

In this paper, we reexamine the definition of relative localization and define time-bounded essential relative localization, a novel problem that captures the minimum amount of time required by localization before it can be integrated with regular payload transmissions. Based on the definition, we present the first rigorous study on the essential localizability of a wireless sensor network within a given time bound. We also propose an efficient distributed algorithm to perform time-bounded essential localization over a sensor network.

For physical localization in a sensor network, there must exist multiple anchor nodes that are capable of acquiring their physical locations from outband channels (e.g., through a GPS module). Intuitively, if the number of anchors is large enough, the network can always be localized in any time bound. However, in practice there can be only a small number of anchor nodes because of the high cost. Thus, in analogy to essential relative localization, we define the problem of time-bounded essential physical localization and analyze its complexity. Furthermore, we show that our distributed algorithm for essential relative localization can also be used to solve the problem of essential physical localization in polynomial time.

The rest of this paper is organized as follows. In Section II, we introduce the preliminaries for localization in wireless sensor 


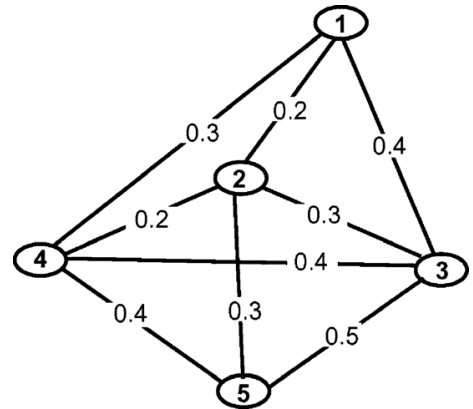

Fig. 1. Example graph modeling a sensor network of five nodes, with the real numbers associated with the edges being the range distances.

networks. The problem of time-bounded essential (relative and physical) localization is defined in Section III. Its complexity from a centralized view is analyzed in Section IV. Section V describes our distributed algorithm for time-bounded essential localization. Section VI presents our simulation evaluation of the proposed algorithm. We briefly review the related work in Section VII, followed by the conclusion in Section VII.

\section{PRELIMINARIES}

\section{A. Graph Model and Basic Notations}

Graph Model: We model a sensor network as a graph $G(V, E)$ in which each vertex $v \in V$ represents a sensor. For the purpose of this paper, we assume all sensors to have an equal communication range. Thus, we consider the graph to be undirected, with an edge $e=(i, j)$ between two vertices ${ }^{1} i$ and $j$ iff their corresponding sensors are within range to communicate with each other.

Fig. 1 depicts an example of such a graph model, which we shall use as a running example. There are five nodes in the figure connected by nine edges. Nodes $1-4$ are interconnected with each other, while 5 is connected with 2-4 only.

Relative and Physical Locations: In general, the location of a sensor node in a $d$-dimensional space can be specified by a $(d+1)$-dimensional vector

$$
\left(\mathrm{ID}, t_{1}, \ldots, t_{d}\right)
$$

where ID is a unique identifier of the $d$-dimensional coordinate system, and $t_{i}$ is the corresponding coordinate of the sensor node at the $i$ th dimension. For the sake of simplicity, we consider all sensors to be distributed in a two-dimensional space and denote the relative location of each sensor by (ID, $x, y$ ). Without causing ambiguity, we denote the physical location of a sensor by $(x, y)$.

Round of Communications: Finally, we define the granularity of time for evaluating the efficiency of a localization algorithm over a wireless sensor network. In particular, we define a round of communications as the amount of time required for a sensor with a degree deg to broadcast a message of length $O(\mathrm{deg})$ to all its neighbors and to receive a message of length $O(\operatorname{deg}(i))$ from each of its neighbors, where $\operatorname{deg}(i)$ is the degree of the $i$ th neighbor.

\footnotetext{
${ }^{1}$ In this paper, we use sensor, node, sensor node, and vertex interchangeably
}

TABLE I

Distance Table of Sensor $i$ With $\operatorname{deg}(j)$ Neighbors $j_{1}, \ldots, j_{\operatorname{deg}(j)}$

\begin{tabular}{|c|c|}
\hline NodeID & Distance \\
\hline$j_{1}$ & $d\left(i, j_{1}\right)$ \\
\hline$j_{2}$ & $d\left(i, j_{2}\right)$ \\
\hline$\ldots$ & $\ldots$ \\
\hline$j_{\operatorname{deg}(j)}$ & $d\left(i, j_{\operatorname{deg}(j)}\right)$ \\
\hline
\end{tabular}

\section{B. Multilateration-Based Localization}

Forming the basic primitive of our study is the multilateration-based localization technique-i.e., the ability of each sensor to measure its distance with the neighboring sensors and to determine its relative position based on the measured distances. For the purpose of this paper, we do not consider the details of lateration (interested readers are referred to [10] for the details on the principles of lateration), but instead formulate it as a preprocessing stage with the following two steps.

- In the first step, each sensor measures its distance to each of its neighbors and stores the measured distances in a distance table as depicted in Table I. Note that $d(i, j)$ is the distance between sensors $i$ and $j$. Also in this step, each sensor broadcasts its distance table (to all of its neighbors). Thus, in one round of communications, each sensor $i$ broadcasts a message of length $O(\operatorname{deg}(i))$, where $\operatorname{deg}(i)$ is the degree of node $i$. For example in Fig. 1, node 5 broadcasts the following message: $\{(5,0),(2,0.3),(3,0.5),(4,0.4)\}$, which is received by nodes $2-4$.

- In the second step, each sensor computes its relative position to its neighbors based on its own distance table and the distance tables received from the neighboring nodes. To understand how such a positioning process works, consider a sensor node $i$. Let $N(i)$ be the set of neighboring sensors of $i$. If three nodes $j, k, h \in N(i) \cup\{i\}$, where $j \neq k \neq h$, are interconnected with each other ${ }^{2}$ and noncollinear, then $i$ has the knowledge of $d(j, k), d(j, h)$, and $d(k, h)$ after receiving all broadcast messages. Based on such information, $i$ can construct an orthogonal coordinate system as follows. First, it sorts $j, k$, and $h$ in an increasing order of their IDs. Without loss of generality, let such an order be $\langle j, k, h\rangle$. Then, it constructs a coordinate system by assigning the location of $j$ to $(0,0)$, the location of $k$ to $(d(j, k), 0)$, and the location of $h$ to $(d(j, h) \times \cos (\theta), d(j, h) \times \sin (\theta))$, where $\theta$ is the angle formed by the edges $(j, k)$ and $(j, h)$. Note that $\theta$ can be obtained through the Law of Cosines, and that the constructed coordinate system is unique in 2-D because $j, k$, and $h$ are noncollinear. In the example shown in Fig. 1, node 5 is capable of constructing a coordinate system under which the coordinates of $3-5$ are $(0,0),(0.4,0)$, and $(0.3125,0.3903)$, respectively. This step incurs no message exchange, and the storage overhead of each sensor $i$ is $O\left(\operatorname{deg}^{2}(i)\right)$.

In multilateration-based localization, each localized node ${ }^{3}$ should broadcast its position to help its neighbors' localization process. Therefore, one round of communication refers to the total time required by the localized nodes to broadcast their

${ }^{2}$ Note that one of $j, k$, or $h$ might be $i$.

${ }^{3} \mathrm{~A}$ localized node is one whose position is available. 


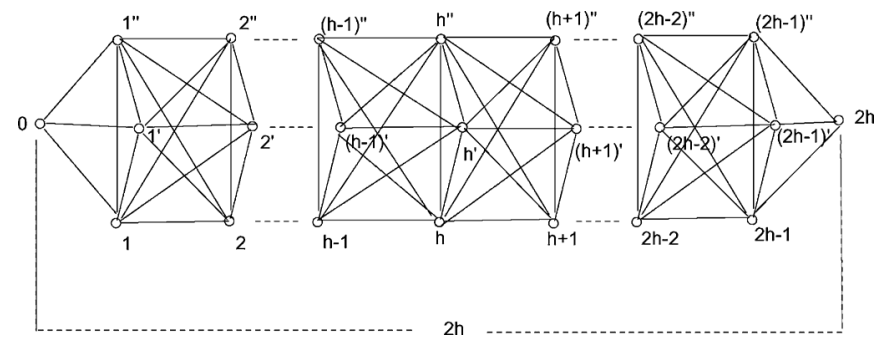

Fig. 2. Example network localizable with one round of communications.

positions and to receive their localized neighbors' broadcasts. The to-be-localized nodes, on the other hand, have to wait for one or more rounds in order to get the position information of three noncollinear neighbors. A to-be-localized node becomes a localized one after obtaining the distance information to three localized neighbors. We employ the number of communication rounds as a metric to quantify the time required to localize the network.

\section{TIME-Bounded EsSEnTIAL LocALIZATION}

In this section, we define a new problem of time-bounded essential localization. In particular, we first explain the deficiency of the traditional evaluation on the overhead of localization time, and then formally define two variants of the time-bounded essential localization for relative and physical positioning.

\section{A. Objective of Essential Localization}

Traditionally, a relative localization process is considered accomplished only if all sensors are positioned in the same coordinate system. Nonetheless, we argue that this is not a proper definition for evaluating the overhead of localization because part of such a process can be seamlessly integrated into the subsequent transmissions of valuable payload information, incurring little extra overhead.

To understand why, consider an example of relative localization as depicted in Fig. 2. With the traditional definition, at least $h$ rounds of communications are required for localization because the radius of the network is $h$. For example, to localize the network under the coordinate system $\left\langle h, h^{\prime}, h^{\prime \prime}\right\rangle$, which is noted as $\langle h\rangle$ for short, $h$ rounds of flooding are required to send the information of $\langle h\rangle$ to the nodes 0 and $2 h$, the two farthest nodes from $\langle h\rangle$. Moreover, the results are the same even if each node starts localizing by constructing a $\underline{L}$ ocal $\underline{C}$ oordinate $\underline{S}$ ystem (LCS) itself instead of waiting for the information from $\langle h\rangle$. The reason is that the traditional definition requires all the nodes to be localized in the same coordinate system. In other words, the nodes' positions under their LCSs need to be transformed to their corresponding positions under a unique global coordinate system. To transform between two 2-D LCSs, a sufficient and necessary condition is that at least three noncollinear nodes are aware of their positions in both LCSs. As a result, each node needs to wait for the information to perform the coordinate system transformation, which also requires at least $h$ rounds of flooding to transform all LCSs to a unique global coordinate system for the example in Fig. 2.
In practice, however, one round of communications might be sufficient for the network shown in Fig. 2. Consider the case where sensor 0 is supposed to transmit a message $M$ as well as its own position $\left(x_{0}, y_{0}\right)$ to node $2 h$ (which can be either a sensor or a sink). Suppose that the routing protocol transmits $M$ through $1,2, \ldots, h, h+1, \ldots, 2 h-1,2 h$. Note that the ultimate objective of localization is for node $2 h$ to be able to understand the locations of 0 and itself in the same coordinate system. This objective can be achieved with exactly one round of localization communications plus coordinate transformations during the regular payload message transmissions.

Let us take a look at the details. In the preprocessing stage, nodes $i, i^{\prime}$, and $i^{\prime \prime}$, where $0<i<2 h$, select the coordinate system $\langle i\rangle$ and localize themselves and their direct neighbors in $\langle i\rangle$. Nodes 0 and $2 h$ localize themselves in $\langle 1\rangle$ and $\langle 2 h-1\rangle$, respectively. Then, each sensor broadcasts its selected coordinate system as well as the coordinates of itself and its neighbors in the selected coordinate system.

Clearly, neither node 0 nor node $2 h$ can position each other in the same coordinate system after this single round of communications. Nonetheless, all future steps needed for localization can be embedded into the actual transmissions of the payload information without incurring any additional communication overhead.

After this single round, node 0 positions itself in the coordinate system $\langle 1\rangle$. Let $\mathrm{LCS}_{1}$ be the ID of this coordinate system and $\left(x_{0}^{1}, y_{0}^{1}\right)$ be the coordinates of node 0 in $\mathrm{LCS}_{1}$. What node 0 sends to node 1 during the payload transmission is

$$
\left\langle M,\left(x_{0}^{1}, y_{0}^{1}\right)\right\rangle .
$$

Note that the length of this message is the same as that when 0 knows its position in a global coordinate system.

After receiving $\left(x_{0}^{1}, y_{0}^{1}\right)$, the processing by node 1 is as follows. First, from the information it learns during the preprocessing step, node 1 is aware of the coordinates of $2,2^{\prime}, 2^{\prime \prime}$ in $\mathrm{LCS}_{1}$. Since during the single round of communications, node 2 has broadcast the positions of itself, $2^{\prime}$, and $2^{\prime \prime}$ in the coordinate system $\mathrm{LCS}_{2}$, node 1 also knows the positions of $2,2^{\prime}, 2^{\prime \prime}$ in $\mathrm{LCS}_{2}$. This enables node 1 to construct a linear transformation between the two coordinate systems.

Now what node 1 does is to transform the location of node 0 from $\mathrm{LCS}_{1}$ to $\mathrm{LCS}_{2}$, and then transmit $\left\langle M,\left(x_{0}^{2}, y_{0}^{2}\right)\right\rangle$ to node 2 , where $\left(x_{0}^{2}, y_{0}^{2}\right)$ are the coordinates of node 0 in $\mathrm{LCS}_{2}$. Again, the message length remains the same as if a global coordinate system exists. We can derive in analogy that the final message received by node $2 h$ is $\left\langle M,\left(x_{0}^{2 h-1}, y_{0}^{2 h-1}\right)\right\rangle$. Since node $2 h$ can also infer its own location in $\mathrm{LCS}_{2 h-1}$ based on the information it receives during the preprocessing stage, the ultimate objective of relative localization is achieved-i.e., node $2 h$ learns the locations of both 0 and itself in the same coordinate system.

One can see from this example that it is unfair to attribute $O(h)$ rounds of communications to the relative localization process (as in the traditional definition) because the communication overhead incurred by localization is only one round - after which localization incurs only computation overhead for coordinate transformation. Note that the time taken by one round of communications is defined to be the maximum 


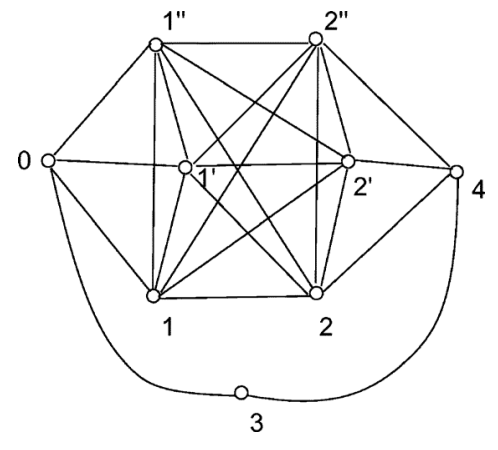

Fig. 3. Example network that requires two rounds of communications in order for the packets to be routed from 0 to 4 via the shortest path.

amount of time for a node to broadcast its positioning information and to receive its neighbors' broadcastings.

It is important to understand that such an improvement depends on the network topology, the selected coordinate system, and the employed routing protocol. For example, the communication overhead of localizing the network shown in Fig. 3 includes two rounds of communications when packets are routed from node 0 to 4 via the shortest path that passes node 3 when $\mathrm{LCS}_{1}$ and $\mathrm{LCS}_{2}$ are selected to localize nodes $0,1,1^{\prime}, 1^{\prime \prime}$ and nodes $2,2^{\prime}, 2^{\prime \prime}, 4$, respectively, in the preprocessing stage. After the first round of broadcasting, node 0 is aware of the positions of nodes $1,1^{\prime}, 1^{\prime \prime}$ in $\mathrm{LCS}_{1}$, but not in $\mathrm{LCS}_{2}$. To compute their positions in $\mathrm{LCS}_{2}$, another round of communications is needed. In other words, node 0 needs two rounds of communications to get sufficient information for $\mathrm{LCS}_{1}$ and $\mathrm{LCS}_{2}$ transformation. Note that node 3 is not localizable, and that only one round of communications is needed if packets are routed through 1 and 2 to reach 4.

From the above discussions, we argue that the objective of a localization process should be to provide each node sufficient information such that any future transmissions of the payload information can automatically integrate the locations of the source and destination into the same coordinate system. This is a weaker requirement than having all sensors share the same coordinate system immediately after the localization process. We formally define this new objective as follows.

Definition 3.1: (The Objective of Essential Relative Localization): Essential relative localization is accomplished if and only if for any pair of nodes $i, j$ in the network, there exist a sequence of coordinate systems $\operatorname{LCS}_{i_{1}, j_{1}, k_{1}}, \ldots, \mathrm{LCS}_{i_{h}, j_{h}, k_{h}}$ such that

- $i$ can position itself under $\operatorname{LCS}_{i_{1}, j_{1}, k_{1}}$;

- $j$ can position itself under $\mathrm{LCS}_{i_{h}, j_{h}, k_{h}}$;

- for any $s \in[1, h-1]$, there exists a sensor $u$ such that $u$ is capable of transforming a position between $\operatorname{LCS}_{i_{s}, j_{s}, k_{s}}$ and $\mathrm{LCS}_{i_{s+1}, j_{s+1}, k_{s+1}}$.

The key idea of essential localization is to avoid the unnecessary communications for localization. The objective of essential localization is quite broad. Some nodes could be essentially localizable even if they cannot be localized in any LCS after $k$ rounds of communications. Fig. 4 shows such an example, where the network $\mathrm{N}$ is essentially localizable after $k$ rounds of communications, but the outside node 1 , which does not belong to $\mathrm{N}$, cannot be localized by any of the LCSs in $\mathrm{N}$ because its neighbors have not yet been localized in a global coordinate

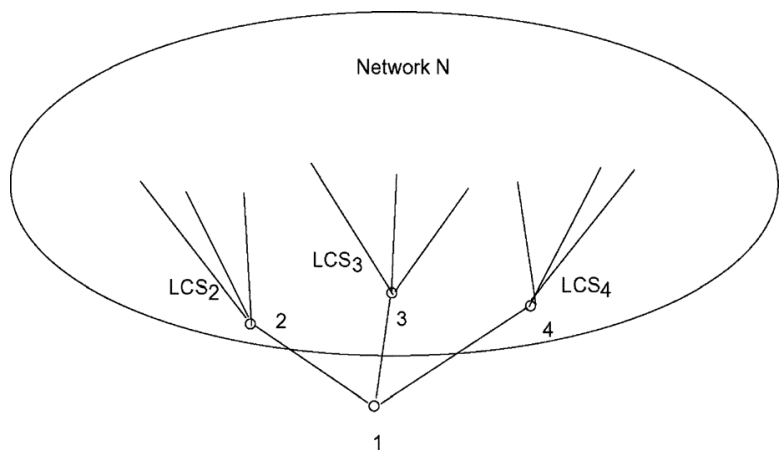

Fig. 4. Network that is essentially localizable with an essentially localizable outside node 1 .

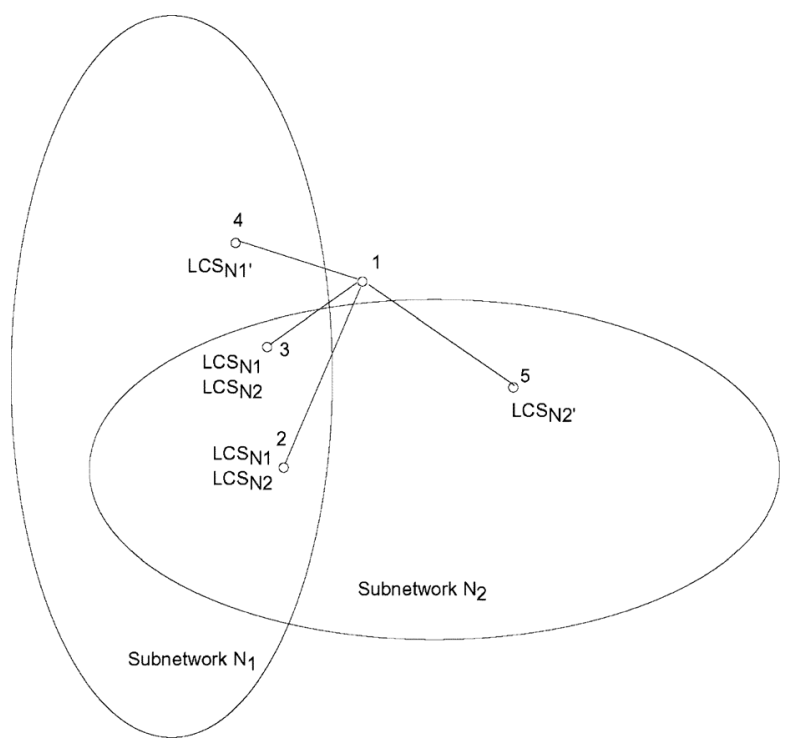

Fig. 5. Example essentially localizable network with subnetworks $N_{1}$ and $N_{2}$ and an essentially localizable outside node 1.

system. However, as $\mathrm{N}$ is essentially localizable, nodes $2-4$ will be eventually localized in a global coordinate system, and thus node 1 will become localizable. Therefore, we mark the network shown in Fig. 4 (the network $\mathrm{N}$ plus the outside node 1) as $k$-round essentially localizable.

Furthermore, outside nodes that are essentially localizable can also contribute to the essential localizability of the whole network. In Fig. 5, $\mathrm{N}_{1}$ and $\mathrm{N}_{2}$ are two essentially localizable subnetworks, and node 1 is an outside node of both $\mathrm{N}_{1}$ and $\mathrm{N}_{2} \cdot \mathrm{N}_{1}$ and $\mathrm{N}_{2}$ can be eventually localized in $\mathrm{LCS}_{\mathrm{N}_{1}}$ and $\mathrm{LCS}_{\mathrm{N}_{2}}$, respectively. Since node 1 has three neighbors in each of $\mathrm{N}_{1}$ and $\mathrm{N}_{2}$, it is essentially localizable in both $\mathrm{LCS}_{\mathrm{N}_{1}}$ and $\mathrm{LCS}_{\mathrm{N}_{2}}$. Then, the whole network (the union of $\mathrm{N}_{1}, \mathrm{~N}_{2}$, node 1 , and the associated edges) is also essentially localizable because nodes 1-3 can be eventually localized in both $\mathrm{LCS}_{\mathrm{N}_{1}}$ and $\mathrm{LCS}_{\mathrm{N}_{2}}$, which implies that the two LCSs are mutually transformable.

\section{B. Formal Definitions}

Based on the objective of essential relative localization, we shall define the concept of $k$-round essential localization and the problem of time-bounded essential localization for relative and physical positioning. 
Definition 3.2: (k-Round Essential Localization): In $k$-round essential localization, sensors intend to localize themselves under their local coordinate systems during the first $k$ rounds of communications; after that, they can localize themselves only through transferring positioning information among the available LCSs.

Definition 3.3: (Time-Bounded Relative Localization): A wireless sensor network is $k$-round localizable if and only if there exists an algorithm that accomplishes the essential relative localization within $k$ rounds of communications.

This definition reflects our novel view of the relative localization: It is accomplished within a bounded time, i.e., after $k$ rounds of communications, the subsequent payload transmissions can be used to transform the positions of communicating nodes into the same coordinate system without extra communication overhead.

Note that this definition may not be applicable to all sensor networks-for a sensor network whose topology is a disconnected graph, it is not localizable even when $k \rightarrow \infty$ because the distance between two disconnected sensors can never be determined.

Such a situation changes in physical localization, however, because anchor nodes can be placed into isolated components of a network for positioning all sensors in the physical coordinate system. In an extreme case, if each sensor is within the communication range of three anchors, the preprocessing step is sufficient for positioning every sensor. Thus, the definition of localizability for physical localization must involve both the number of communication rounds required for localization and the number of anchor nodes deployed to a network.

Definition 3.4: (Time-Bounded Physical Localization): A wireless sensor network is $k$-round localizable with $\ell$ anchors if and only if there exists a location configuration of $\ell$ anchors $\alpha_{1}, \ldots, \alpha_{\ell}$ and a localization algorithm that terminates within $k$ rounds of communications such that for any sensor $i$, there exist at least three anchors $\alpha_{u}, \alpha_{v}, \alpha_{w}$, and for each of these anchors, say $\alpha_{u}$, there exist a sequence of coordinate systems $\mathrm{LCS}_{i_{1}, j_{1}, k_{1}}, \ldots, \mathrm{LCS}_{i_{h}, j_{h}, k_{h}}$ satisfying the following:

i) $i$ can position itself under $\operatorname{LCS}_{i_{1}, j_{1}, k_{1}}$;

ii) $\alpha_{u}$ can position itself under $\operatorname{LCS}_{i_{h}, j_{h}, k_{h}}$;

iii) for any $s \in[1, h-1]$, there exists a sensor $v$ such that $v$ is capable of transforming a position between $\mathrm{LCS}_{i_{s}, j_{s}, k_{s}}$ and $\mathrm{LCS}_{i_{s+1}, j_{s+1}, k_{s+1}}$.

One can see from the definition that if a sensor network is $k$-round localizable with three anchors, it must be $k$-round localizable for relative localization. To understand why, we observe that the sequence of coordinate systems specified in Definition 3.4 is invertible. With three anchors, for every pair of sensors $i$ and $j$, there must exist an anchor, say $\alpha_{1}$, with which both $i$ and $j$ are connected through a sequence of coordinate systems. The concatenation of these two sequences defines a sequence that connects $i$ and $j$ and thus fulfills the localizability requirement for relative localization.

\section{COMPleXity ANAlysis FOR TIME-BOUnded LOCALIZATION}

\section{A. Preliminaries}

In order to analyze the complexity of localizing a given network in a given time-bound, we assume that the following parameters are available: the time bound $k$, the network size $N$, the network diameter $\alpha$, and the maximum node degree $\beta$. We start our analysis from a sensor's local $k$-hop graph, the induced graph of the sensor's neighbors within $k$-hop distance. Lemma 4.1 claims that a sensor's local $k$-hop graph covers all the possibilities that can make the sensor localizable in $k$ rounds of communications.

In a graph, there might exist multiple anchors and multiple possible LCSs because every three mutually connected nodes can construct an LCS. The localization process can start from a subset of anchors and/or a subset of LCSs. We denote the set of the selected anchors and/or LCSs as a combination. In the $(d+1)$-lateration localization for $d$-dimensional space, a necessary and sufficient condition for a node's localizability is that the node has $(d+1)$ direct localizable neighbors to which it has the distance information. Then, to localize the node in $k$ rounds of communications, those $(d+1)$ direct neighbors should be localized within $(k-1)$ rounds of communications. With the same reasoning, we claim that any of the node's $h$-hop neighbors could contribute to the node's localizability only if it can be localized within $(k-h)$ rounds of communications. Therefore, the anchors or the local coordinate systems that are $(k+1)$-hop away from the node do not help with the node's localizability in $k$ rounds of communications.

Lemma 4.1: All the combinations of the anchors and/or the local coordinate systems that can cooperate to localize a node under some LCS in $k$ rounds of communications reside in the node's local $k$-hop graph.

Proof: The statement is true for the combination of anchors because anchors located at more than $k$ hops away cannot contribute to the localization of the node in $k$ rounds of communications

Let $C$ be a node that is more than $k$ hops away from the to-belocalized node $S$. Assume that $C$ participates in constructing a local coordinate system $\operatorname{LCS}_{c}$ that contributes to node $S$ 's localization in $k$ rounds of communications. Then, there must exist a node $T$ that can be localized in $\mathrm{LCS}_{c}$, and $T$ is essential to localize the node $S$ in $k$ rounds of communications. Without loss of generality, we assume that $T$ is localized in $\mathrm{LCS}_{c}$ at the $t$ th round of communications. This means that $T$ is at most $(k-t)$ hops away from $S$ in order to localize $S$ in $k$ rounds of communications, and that $T$ is at most $t$ hops away from $C$ in order to be localized by $\mathrm{LCS}_{c}$ at the $t$ th round of communications. Therefore, the hop distance between $C$ and $S$ is at most $k$, which contradicts our assumption. Thus, the statement is true for the combination of local coordinate systems.

For a given network $G(V, E)$, the number of nodes in a node's local complete $k$-hop graph is $O\left(\beta^{k}\right)$, where $\beta$ is the maximum node degree. For each node, it can participate in constructing $O\left(\beta^{d}\right)$ number of local coordinate systems in the $d$-dimensional space. Then, there exist in total $O\left(N \times \beta^{d}\right)=H$ number of possible LCSs in the network, where $N$ is the network size. In a node's local $k$-hop graph, there exist $O\left(\beta^{d+k}\right)$ number of possible LCSs; and the number of all possible combinations of the LCSs is $2^{O\left(\beta^{d+k}\right)}=M$. For better elaboration, a combination of the LCSs is denoted as a cooperating LCS (CLCS).

For each CLCS, as the number of communication rounds for localization is bounded by $k$, it takes $O\left(k \times \beta^{k}\right)=P$ time to 
identify the nodes that can be localized under some LCS of the CLCS. Therefore, it takes $O(P \times M \times N)$ time to figure out which node can be localized under which LCS after $k$ rounds of communications. Note that the time complexity is polynomial to $N$.

In the following, we begin with analyzing the complexity of time-bounded relative localization.

\section{B. Complexity of Time-Bounded Essential Relative Localization}

A necessary and sufficient condition for mutually transforming coordinates between two LCSs is that there exist at least $(d+1)$ nodes that are aware of their positions in both LCSs in the $d$-dimensional space. According to Section IV-A, each possible $\mathrm{LCS}_{i}, 1 \leq i \leq H$, can obtain its $A_{i}$, the set of nodes that can be localized under $\mathrm{LCS}_{i}$ in $k$ rounds of communications, in polynomial time. Then, it takes at most $O\left(N \times H^{2}\right)$ time to transform all the LCSs into a locally unique coordinate system (LUCS) through enumeration. Considering the essentially localizable nodes outside the network and their contributions to the network's $k$-round essential localizability, it takes at most $O\left(N^{2}\right) \times O\left(N \times H^{2}\right)$ time in total to eventually transform all the LCSs into the LUCS. Note that there might exist multiple LUCSs when the network is not essentially localizable in $k$ rounds of communications. We call each LUCS an isolated LCS island. An isolated LCS island is a set of mutually transformable LCSs. Any two LCSs in two different islands are not transformable to each other.

Therefore, the complexity of checking whether a given network can be essentially relatively localized in $k$ rounds of communications is polynomial to $N$, which is the number of nodes in the network. Let $\alpha$ be the network diameter. It takes at most $T=O(N \times H \times \alpha) \times O\left(N^{2}\right) \times O\left(N \times H^{2}\right)$ time, which is also polynomial to $N$, to figure out whether the network is localizable in $k$ rounds of communications and to obtain the minimum $k$ that guarantees the network's localizability.

\section{Complexity of Time-Bounded Essential Physical Localization}

Following the results presented in Section IV-B, we study the complexity of time-bounded physical localization in this section. According to Definition 3.4, we should address the following questions for time-bounded physical localization.

1) Given a network $G$ and the deployed anchors, is the network essentially physically localizable in $k$ rounds of communications?

2) Given a network $G$ and the deployed anchors, is the network localizable? If yes, what is the minimum required communication rounds $k$ ?

3) Given a network $G$ and $k$, how should the anchors be deployed such that the network is localizable in $k$ rounds of communications and the number of anchors is minimized?

To answer these questions, we construct a new graph $G_{T}\left(V_{T}, E_{T}\right)$, where $V_{T}=V_{U} \cup V_{N} \cup V_{A}$ and $E_{T}=$ $E_{U N} \cup E_{U A}$, with $V_{U}, V_{N}, V_{A}, E_{U N}$, and $E_{U A}$ being defined as follows:

- LUCS set $V_{U}$, where each node represents a locally unique coordinate system;
- to-be-localized node set $V_{N}$, where each node represents a to-be-localized node in the network $G$;

- candidate anchor set $V_{A}$, where each node represents a potential anchor node in the network $G$;

- dominating edge set $E_{U N}$, where each edge joints a LUCS node in $V_{U}$ and a node in the to-be-localized node set $V_{N}$ if the node in $V_{N}$ can be localized under the LUCS in $k$ rounds of communications;

- anchor edge set $E_{U A}$, where each edge joints a LUCS node in $V_{U}$ and a node in the candidate anchor set $V_{A}$ if the node in $V_{A}$ can be localized under the LUCS in $k$ rounds of communications.

Note that it takes polynomial time to construct the graph $G_{T}$ according to the analysis in Section IV-B. For the first question, it is equivalent to the problem of finding whether there exists a set $V_{U}^{\prime} \subseteq V_{U}$ such that each node in $V_{N}$ is dominated by at least one node in $V_{U}^{\prime}$, and that each node in $V_{U}^{\prime}$ connects to at least three nodes in $V_{A}$. Obviously, this problem can be easily solved in polynomial time. To answer the second question, we need to construct $G_{T}$ and to check whether the network $G$ is essentially physically localizable in $k$ rounds of communications for each possible $k$. According to the analysis presented in Section IV-B, $k$ is upper-bounded by $O(N \times H \times \alpha)$. Therefore, the time complexity of answering the second question is also polynomial.

For the third question, the solution set $V_{A}^{\prime} \subseteq V_{A}$ should simultaneously satisfy the following three requirements.

- For any $V_{U}^{\prime} \subseteq V_{U}$, each node in $V_{U}^{\prime}$ is dominated by at least three nodes in $V_{A}^{\prime}$.

- Each node in $V_{N}$ is dominated by at least one node in $V_{U}^{\prime}$.

- $\left|V_{A}^{\prime}\right|$ is minimized.

It is easy to conclude that the hitting set problem [11] is a degeneration of the above problem. Therefore, generally speaking, the third question is NP-hard. Intuitively, it is related to the dominating set and connected dominating set problems, with both being proved to be NP-hard [12], [13]. There exist many efforts [14]-[17] to construct a connected dominating set in sensor networks. A survey of these algorithms can be found in [18]. Nevertheless, none of the proposed algorithms could be applied to solve the time-bounded essential localization problem because they focus on the 1-hop 1-dominating set problem. Moreover, the practical constraint also makes it impossible to construct the graph $G_{T}$ in real-world implementations. On the other hand, the time-bounded essential localization problem is more likely to be related to the $k$-hop $(d+1)$-dominating set problem, in which a node should either be a dominator or be dominated by $(d+1)$ dominators within $k$ hops away. Distributed algorithms for the k-hop 1-dominating set problem, which is proved to be NP-hard [12], are reported in [19] and [20]. To our knowledge, none of the existing works focuses on the $k$-hop $(d+1)$-dominating set problem for a positive integer $d$. Furthermore, solutions for the $k$-hop $(d+1)$-dominating set problem can only produce necessary solutions for the time-bounded localization problem because they can at most guarantee $k$-hop $(d+1)$-dominating graphs, which are insufficient for localizability as reported in Section VII.

In our investigation, the most related research to the timebounded essential physical localization problem is the Fast Information Propagation problem proposed in [21]. This problem 


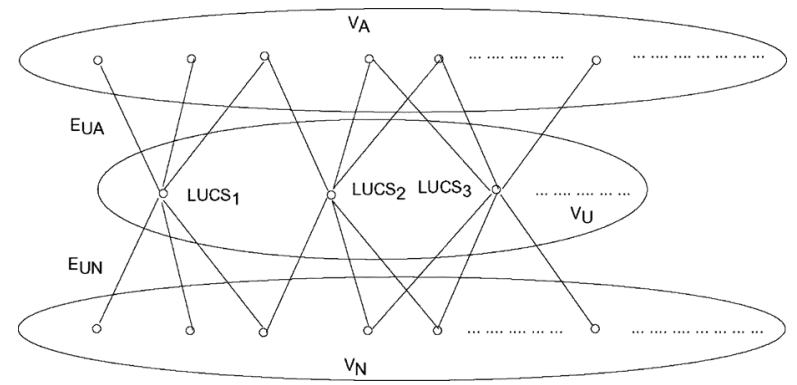

Fig. 6. Example $G_{T}$.

intends to extract the minimum size of nodes for initial activation such that information could be sent to all the nodes in the given social network within a bounded time. It has been proved [21] that the fast information propagation problem is NP-hard when the time bound (number of hops) is 1 and the number of dominators is $\beta / 2$, which is half of the maximum node degree. An approximate algorithm is also proposed in [21]. Under the same conditions, Zhu et al. [22] prove that the fast information propagation problem is APX-hard whether the initial active nodes are connected or not. Although the fast information propagation problem is a good match to our time-bounded essential localization problem, the 1-hop constraint makes the currently focused problem degenerate to be a variation of the dominating set problem. Therefore, as stated above, no existing result can be applied to our general time-bounded essential localization problem.

\section{Analysis on Optimal Anchor Selection for Time-Bounded Physical Localization}

In this section, we study the problem of how to select the minimum number of nodes as anchors so that the network can be physically localized in a given time bound. Note that we use the same notations as those in Section IV-C. Fig. 6 shows an example graph $G_{T}$, where $\left|V_{U}\right| \leq H$, and $\left|V_{A}\right|=\left|V_{N}\right|=N$. Obviously, $V_{A}$ and $E_{U A}$ are the reflections (copies) of $V_{N}$ and $E_{U N}$, respectively, when each node in $V_{N}$ can be an anchor candidate. As indicated in Sections IV-B and IV-C, given the number of rounds $k$, the graph $G_{T}$ can be constructed in polynomial time. Additionally, according to the objective stated in Section III-A, each LUCS in $V_{U}$ has at least three neighbors in $V_{N}$. Moreover, any two LUCS in $V_{U}$ should have at most two common neighbors in $V_{N}$ since otherwise they should be merged into one LUCS.

To physically localize the network, we first identify those isolated nodes that cannot be localized by any LUCS. These isolated nodes have to be chosen as anchors. Then, we look for a minimum subset of $V_{A}$ such that the nodes in $V_{U}$, which are three-dominated by the selected subset, can dominate all the nodes in $V_{N}$. We set the nodes in the selected subset of $V_{A}$ as anchors.

To find the minimum subset, an intuitive question to ask is the following: Are all the LUCSs essential to localize the network? In other words, does each LUCS localize some nodes that cannot be localized by other LUCSs? If the answer is Yes, the problem can be degraded to the three-dominating set problem. Unfortunately, the answer is No because the nodes, which can be localized by $\mathrm{LUCS}_{2}$, can also be localized by either $\mathrm{LUCS}_{1}$ or $\mathrm{LUCS}_{3}$ as shown in Fig. 6. A possible solution to this problem is to employ enumeration on $V_{A}$, which has a time complexity of $2^{N}$.

Our next goal is to find the lower bound of the minimum number of anchors to physically localize a network with diameter $\alpha$ in $k$ rounds, which is denoted as $N_{A}$. Suppose that each node can be localized in some LUCSs. Then, the minimum number of LUCSs that can localize the whole network is lower-bounded by $H^{\prime}=\frac{\alpha^{2}}{k^{2}}$. This means that $H^{\prime}$ is the number of essential LUCSs to cover the whole network in $k$ rounds. As any two of these essential LUCSs can share at most two common neighbors in $V_{N}$, we have

$$
C_{\underline{N_{A}}}^{3}=H^{\prime}
$$

which implies that any three nodes in the minimum required anchor set, whose cardinality is $N_{A}$, can construct an essential LUCS. Then, we can obtain the theoretical lower bound of the minimum number of anchors by solving (2), with $N_{A}$ being the only unknown.

Theorem 4.1: Given a network with diameter $\alpha$, the lower bound of the number of required anchors, which can cooperatively localize the network in $k$ rounds, is $N_{A}=\max \left\{3,\left\lceil\operatorname{root}_{\min }^{\text {positve }}\right\rceil\right\}$.

Note that root min $_{\text {pine }}^{\text {posive }}$ is the minimum positive root of (2).

Although there exist some greedy methods for finding approximate solutions, we simply skip the discussions because they are centralized, which makes them inapplicable in wireless sensor networks. In the next section, we propose a distributed approximate solution for the essential localization problem.

\section{Distributed Algorithm}

In this section, we describe a distributed algorithm for timebounded essential localization. From an algorithmic perspective, the objectives of essential localization for both relative and physical positioning are the same-i.e., to maximize the number of localized nodes at any given time bound.

\section{A. Algorithm Design}

The algorithm is depicted in Algorithm 1. The only input is the time bound-i.e., the maximum number of communication rounds that can be consumed by the localization process. The algorithm requires each sensor node to maintain the following data structures:

- a position table, which stores the positions of all 1- and 2-hop neighbors of the sensor node under all LCSs constructed by the sensor;

- an LCS identification table, which specifies how each LCS is constructed (i.e., the IDs of all the sensors that define the LCS);

- an LCS transformation table, which specifies the transformation between each LCS in the LCS identification table and a base LCS (BLCS). Note that each node has its own BLCS.

The BLCS is an LCS, which is constructed by the sensor itself and two of its direct neighbors, such that the number of localized nodes under this LCS is no less than that by any other 
LCS in the node's 2-hop topology. The BLCS is determined as follows. At each communication round, a sensor broadcasts its 1-hop position table so that its neighbors can update their corresponding 2-hop position tables. Then, based on the 2-hop position table, each sensor constructs its BLCS by finding two of its 1-hop neighbors such that: 1) these two neighbors are within the communication range of each other; and 2) the LCS constructed by the two neighbors and the sensor itself can localize the maximum number of sensors in the 2-hop topology. Note that this step can be achieved by brute-force searching and trilateration as the sensor is aware of its 2-hop topology. The time complexity is $O\left(\beta^{2}\right)$, with $\beta$ being the node degree. Once the BLCS is selected, three steps are performed by the function Update the LCS transformation table: First, a sensor checks its position table to find all the LCSs that can be transformed to the BLCS; second, the sensor transforms all LCSs identified in the first step to the BLCS; finally, the sensor localizes more sensors based on the updated position table. The output of the algorithm includes the following information:

- Num_Island: the number of isolated LCS islands remaining in the network. Num_Island presents how many islands that are not globally transformable in the network if we run the proposed algorithm for an infinite number of rounds.

- Iteration_Bound: the max/min number of communication rounds required to connect all LCSs in every island (as defined above).

- Max_Localized: the number of localized sensors in the isolated LCS island that contains the largest number of localized nodes among all the isolated LCS islands.

- Num_Unlocalized: the number of unlocalized nodes in the network-i.e., the number of nodes that cannot be essentially localized.

- Num_Anchors: the number of anchors required to physically localize the network.

Based on the outputs, a sensor network is $k$-round essentially relatively localizable if Iteration_Bound $\leq k$, Num_Island $=1$, and Num_Unlocalized $=0$. For physical localization, a sensor network is $k$-round localizable when Iteration_Bound $\leq k$. The number of required anchors is calculated by

$$
3 \times \text { Num_Island }+ \text { Num_Unlocalized }
$$

which means that each island has three anchors, and that each unlocalized node is an anchor. Note that (3) is an upper bound of the number of required anchors. As any two islands can have at most two common nodes, the lower bound of the required number of anchors is

$$
2+\mathrm{Num}_{\text {Island }}+\mathrm{Num}_{\text {Unlocalized }}
$$

which implies that all the islands share the same two common nodes that have been chosen as anchors. The output Num Anchors is the upper bound defined in (3).

\section{B. Algorithm Analysis}

In this section, we shall first show that our algorithm is capable of positioning more sensors (through polynomial-time transformations among LCSs) than the traditional technique.
Algorithm 1: Time-Bounded Localization Algorithm

1: iteration $=0$;

2: while iteration $<k$ do

3: $\quad$ iteration $=$ iteration +1 ;

4: $\quad$ Subphase I: Broadcasting and Collecting

5: if the LCS identification table has been updated then

6: $\quad$ Broadcasts the update of the LCS identification table;

7: $\quad$ end if

8: if the position table has been updated then

9: $\quad$ Broadcasts the update of the position table;

10: end if

11: if the LCS transformation table has been updated then

12: $\quad$ Broadcasts the update of the LCS transformation table;

13: end if

14: Collects all the messages from its neighbors;

15: $\quad$ Subphase II: Localization

16: Updates the LCS identification table;

17: Updates the position table;

18: Combines the LCS transformation table with the received messages;

19: Selects a BLCS;

20: $\quad$ Updates the LCS transformation table;

21: while the the position table has been updated do

22: $\quad$ Updates the LCS transformation table;

23: end while

24: end while

25: Outputs the position table and the LCS transformation table;

26:

27: Functions:

28: function UPDATES THE LCS IDENTIFICATION TABLE

29: Combines the LCS identification table with the received messages;

30: end function

31: function UPDATES THE POSITION TABLE

32: Combines the position table with the received messages;

33: Localizes the nodes under LCSs;

34: end function

35: function UPDATES THE LCS TRANSFORMATION TABLE

36: Transforms the transformable LCSs to the BLCS;

37: Transforms the positions in the transformable LCSs to the BLCS;

38: Updates the position table;

39: end function

Then, we investigate the local optimality of our algorithm in selecting a BLCS. We prove that, within a sensor's 2-hop topology, the group of sensors that can be localized by the selected BLCS is a superset of those that can be localized by other LCSs that can also localize the sensor. 


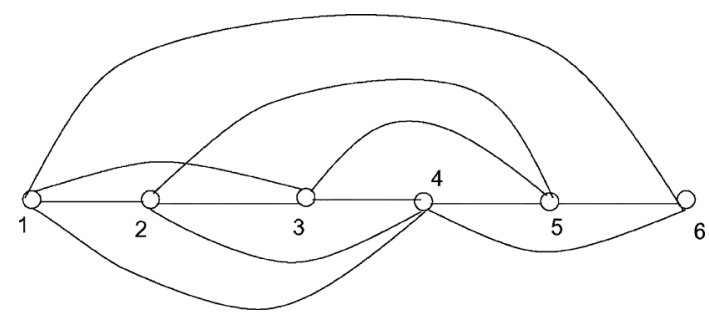

Fig. 7. Example to illustrate the ordered list of the localized nodes in $\mathrm{LCS}_{123}$.

As multilateration is the basic approach adopted by each LCS to localize a network, there exists an order of the localized nodes for each LCS, in which each node except the three origins defining the LCS has at least three ancestor nodes. In other words, following that order, all the nodes can be localized by the three origin nodes of the LCS. However, if randomly selecting three nodes in the ordered list, we cannot guarantee the localizability of their ancestor nodes nor the localizability of all the nodes after them in the list. Note that we require the selected three nodes to be nondegenerative. Fig. 7 shows such an example, where there exist six localized nodes in $\mathrm{LCS}_{123}$, with nodes 1-3 being the three origins. Nodes 4-6 can be localized in the order of $\langle 4,5,6\rangle$ in $\operatorname{LCS}_{123}$, but none of the origins can be localized by them.

Note that if two LCSs can be mutually transformable, the intersection of their ordered lists of the localized nodes should have at least three degenerative nodes. According to the above analysis, it can be concluded that the nodes in the intersection cannot guarantee the localizability of all the nodes in both lists. Therefore, we obtain the following theorem.

Theorem 5.1: Given a network and a set of anchors, the number of localized nodes by applying the proposed algorithm is larger than or equal to the number of localized nodes by applying the traditional multilateration localization method.

Proof: For any node in the ordered list of an LCS, it can obtain its physical position through position transformations when there exist at least three anchors in the list. Starting from these anchors, we can obtain a sublist of the LCS's list through the traditional multilateration localization method. This means that the LCS can localize all the nodes that can be localized by these anchors. Note that the ordered list starting from any anchor must be a sublist of some LCS. However, according to the above analysis, the sublist cannot guarantee the localizability of the two original lists. Therefore, the proposed algorithm can localize all the nodes that can be localized by all the anchors, but not vice versa.

Within the 2-hop topology of a node, there does not exist an edge to connect two 2-hop neighbors of the node because its 1-hop neighbors only broadcast their 1-hop topologies. Intuitively, for a node to construct a local optimal LCS that can localize the maximum number of nodes (including itself) in its 2-hop topology, it is sufficient to consider all the possible three-node combinations as LCS candidates, in which the three nodes should mutually hear each other. The time complexity of this enumeration procedure is $O\left(\beta^{4}\right)$, where $\beta$ is the maximum node degree, because an LCS candidate can include at most one of the node's 2-hop neighbors. Nevertheless, as in- dicated by Theorem 5.2, the time complexity of our proposed BLCS selection method is $O\left(\beta^{2}\right)$. This theorem also proves that the proposed BLCS selection method is sufficient to find a local optimal LCS.

Theorem 5.2: In the proposed distributed algorithm, the selected BLCS is a local optimal LCS within the 2-hop topology of a node.

Proof: In the proposed distributed algorithm, we check all the LCS candidates that are constructed by the node itself and two of its direct neighbors to select a BLCS. We denote this type of LCS candidates as $S(0,1,1)$, where 0 represents the node itself and 1 represents one of its 1-hop neighbors.

A possible LCS candidate can also be constructed by three of the node's 1-hop neighbors. We denote this type of LCS candidate as $S(1,1,1)$, where 1 represents one of the node's 1-hop neighbors. Moreover, a possible LCS candidate can be constructed by one of the node's 2-hop neighbors and two of the node's 1-hop neighbors. We denote this type of LCS candidate as $S(2,1,1)$, where 2 represents one of the node's 2-hop neighbors and 1 represents one of the node's 1-hop neighbors. Our proposed method only needs to check the LCS candidate set $S(0,1,1)$.

Assume that a local optimal LCS is in the candidate set $S(1,1,1)$. As all the three 1-hop neighbors that construct the local optimal LCS can mutually hear each other and the node itself connects to all of them, the LCS constructed by the node and any two of the three 1-hop neighbors can localize the other 1-hop neighbor. Therefore, all the three origins of the local optimal LCS can be localized by the newly constructed LCS that is in the set $S(0,1,1)$. Then, the newly constructed LCS can localize all the nodes that can be localized by the local optimal LCS. Thus, the newly constructed LCS is a local optimal LCS.

Assume that a sensor's local optimal LCS is in the candidate set $S(2,1,1)$. As the local optimal LCS can localize the sensor itself, it must be able to localize at least one more 1-hop neighbor. The LCS, which is constructed by this 1-hop neighbor and the other two 1-hop neighbors in $S(2,1,1)$, can localize the 2-hop neighbors in the set $S(2,1,1)$. Therefore, the newly constructed LCS, which is in the set $S(1,1,1)$, can localize all the three origins of the local optimal LCS. Thus it is a local optimal LCS. According to the analysis of $S(1,1,1)$, there must exist a local optimal LCS that is in the candidate set $S(0,1,1)$.

In conclusion, checking only the candidate set $S(0,1,1)$ is sufficient to find a local optimal LCS within a node's 2-hop topology. This means that our proposed BLCS selection method can find a local optimal LCS in $O\left(\beta^{2}\right)$ time.

Note that the result of Theorem 5.2 cannot hold if the communication edge between two nodes is not bidirectional.

\section{Simulation Evaluation}

In this section, we use MATLAB simulations to evaluate the performance of our proposed algorithm for essential localization.

\section{A. Simulation Settings}

In our simulation, each sensor node runs our proposed localization algorithm. The topology of the sensor network is set 


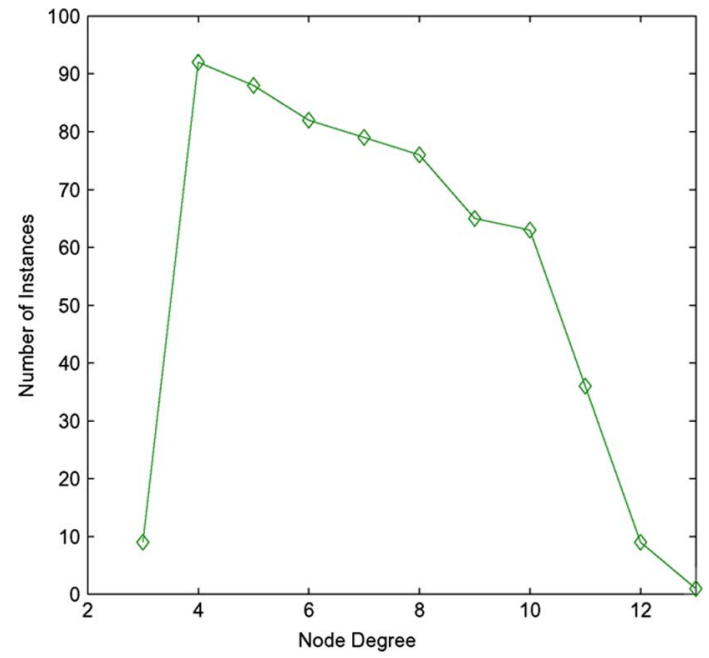

Fig. 8. Number of instances for each node degree.

up as follows. We consider a sensor network consisting of 100 nodes randomly deployed in a two-dimensional square region with a size of $100 \times 100$. We denote the region's border length and the number of sensors in the network by $d=100$ and $N=100$, respectively. Thus, the node density can be approximated by $\frac{N}{d^{2}}$. To control the network average node degree, we vary the node's communication range from $R_{1}$ to $R_{2}$ such that $R_{1}^{2} \times \frac{N}{d^{2}}=1$ and $R_{2}^{2} \times \frac{N}{d^{2}}=4$-i.e., $R_{1}=10$ and $R_{2}=20$. This yields a node degree ranging approximately from 3 to 13 . Note that the transmission ranges are the same for all the nodes at each simulation. The outcomes of all simulations are averaged over 50 i.i.d. random network instances.

\section{B. Simulation Results}

In this section, we report our simulation results to study the time-bounded localization problem. The performance metrics include the Iteration_Bound, which is the minimum number of communication rounds required to connect all the LCSs in any isolated LCS island, the number of isolated LCS islands, the maximum number of localized nodes in the largest isolated LCS island, the number of unlocalized nodes, and the estimated number of anchors required to physically localize the network. Note that the results reported for the node degree $m$ summarize those from $m-1$ to $m$. As the node degree cannot be directly controlled, Fig. 8 presents the number of instances each node degree appears in the simulations. Note that the following reported results are the average of all the instances at its corresponding node degree.

Fig. 9 reports the results regarding how many nodes are unlocalizable, how many nodes can be localized in the largest isolated island, and how many anchors are required to physically localize the network. Generally speaking, all the curves are monotonic from node degree 3 to 12 . An exception appears at node degree 13. The reason lies in that the number of instances at degree 13 is very low as shown in Fig. 8, thus the reported result cannot represent the general property of degree 13 . When the node degree reaches 10 , almost all the nodes become localizable, and about $75 \%$ of the nodes can be localized at the largest island.

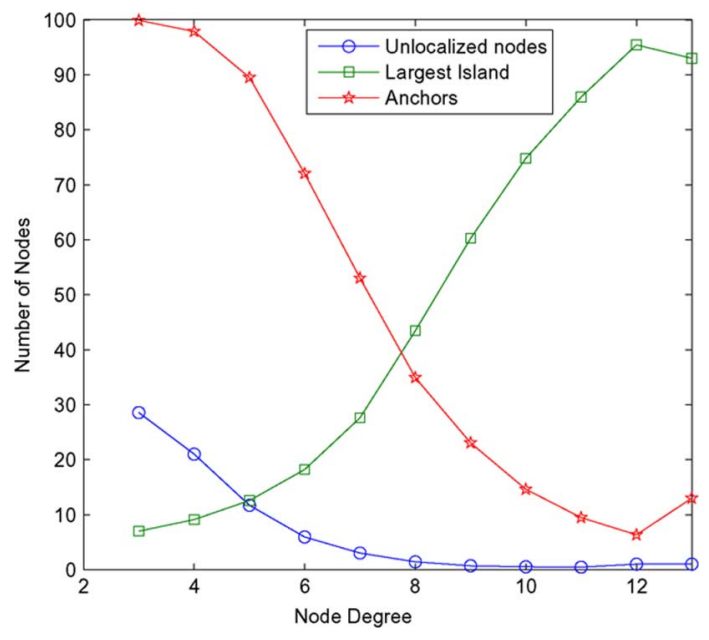

Fig. 9. Numbers of the nodes that are localized in the largest island and that are unlocalized, and the number of anchors required to physically localize the network.

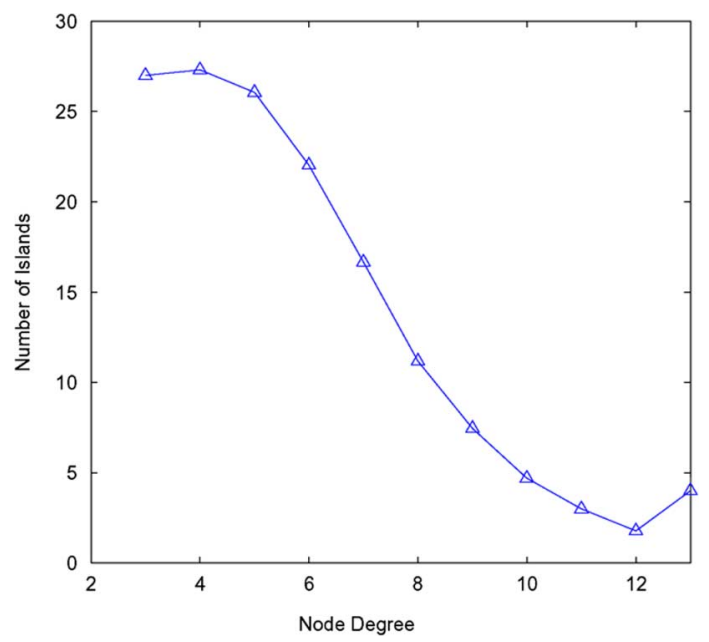

Fig. 10. Number of Isolated Islands.

Fig. 10 reports the number of isolated islands identified by the proposed algorithm versus the node degree. Generally speaking, this is a decreasing curve because the LCSs have more chances to transform to each other when the node degree is larger. An exception appears when the node degree varies from 3 to 4 . A possible reason is that the degree 4 is still a low degree, but more nodes become localizable when constructing more LCSs compared to the degree 3 . The number of islands is relatively high and decreases fast when the node degree is less than 10 . The curve goes down slowly when it passes the point (degree) 10, where the number of isolated islands is about 5 . The exception at node degree 13 is resulted from the low number of instances at degree 13, as elaborated for Fig. 9.

Fig. 11 reports the iteration bounds of the network's localizability for given node degrees under both the newly defined essential localization and the traditional trilateration localization. A maximum localization time represents the worst case among all the instances for the given node degree in the simulations. It is easy to observe that the proposed essential localization algorithm significantly outperforms the trilateration localization in terms of localization time. Note that the standard deviations 


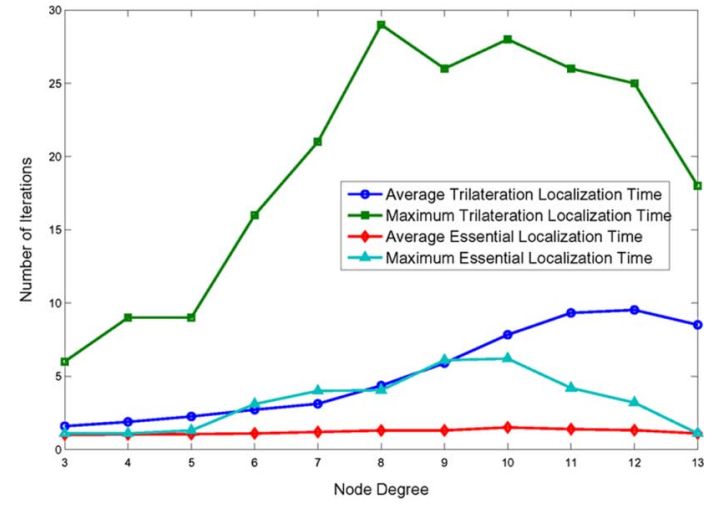

Fig. 11. Iteration bound.

of the reported average essential localization time and the average trilateration localization time are about $62 \%$ and $58 \%$ of the mean values, respectively. It is interesting to observe that the maximum essential iteration bound in the worst case is roughly equal to $\frac{d}{R}$, where $R$ is the communication range. The essential localization bound curve always peaks at the node degree 10 , which demonstrates a change in the trend in both Figs. 9 and 10 , where almost all the nodes become localizable and a large island is formed. A possible reason for the curves' decrease after 10 is that most of the LCSs are mutually transformable and that the nodes' localizability becomes stable. When the node degree is less than 5 , where the number of isolated islands is relatively large, the bound is always 1 . This indicates that all the isolated islands are constructed in 1 iteration because the node degree is too low to satisfy the LCSs' transformation conditions. When the node degree reaches 12 , the maximum bound is also about 1 , where the node degree is large enough to build one big island to localize almost all the nodes (more than 96\%). It is delightful to see that the average bound for all the node degrees is less than 2 , which means that two rounds of communications are sufficient for essentially localizing a large number of nodes.

\section{RELATED WORKS}

The localization problem has been widely investigated in wireless sensor networks for many years, and now it is still a hot research topic. In this section, we first summarize the theoretical results for localization. Then, major existing localization schemes are reviewed under the categories of physical positioning and relative positioning.

From graph theory's point of view, the localization problem asks how to assign a position to each node in the network such that the relations among the edges can be satisfied. Reference [23] claims that the network localization problem is solvable in two-dimensional and three-dimensional space if and only if the network graph is globally rigid. An intuitive description of the rigidity is that the graph cannot flex. A globally rigid graph is unique in the isometry of $d$-dimensional space. Generally speaking, the test for the global rigidity of a graph is NP-hard [24]. A stronger concept is the generic global rigidity. A graph is said to be generic if the set containing the coordinates of all its points is algebraically independent over the rationals. References [25] and [26] study a necessary but

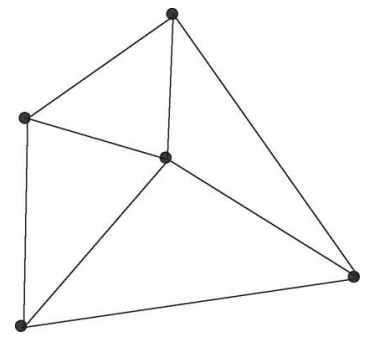

Fig. 12. Wheel graph.

not sufficient condition for the generic global rigidity: If a graph $G$ is generically globally rigid in $d$-dimensional space, then $G$ is redundantly rigid and $(d+1)$-connected. A graph is $k$-connected if it remains connected upon removal of any set of $<k$ vertices. A graph is redundantly rigid in $d$-dimensional space if the removal of any single edge results in a graph that is also generically rigid. This condition has been proved to be both necessary and sufficient in two-dimensional space by [27]. Asix-connected graph is proved to be generically globally rigid in two-dimensional space by [27] as well. However, there exist redundantly rigid and at least $(d+1)$-connected graphs that are not globally rigid when $d>2$ [27].

In general, [23] has proved that the localization problem is NP-hard, and the result holds true even if the network is a unitdisk graph. For example, the wheel graph shown in Fig. 12 is globally rigid but cannot be localized in polynomial time. Fortunately, there exist some kind of graphs that can be localized easily, such as the $(d+1)$-lateration graph. $\mathrm{A}(d+1)$-lateration graph is a graph that has a $(d+1)$-laterative ordering. This is an ordering of all the vertices in the graph such that from any vertex with ordering $j, j>d+1$, there are at least $(d+1)$ edges to the vertices earlier than $j$ in the sequence. The $(d+1)$-lateration graph is globally rigid and has a polynomial-time localization solution in $d$-dimensional space [23]. The $(d+1)$-lateration graph can be localized by the geometrical multilateration method in polynomial time in $d$-dimensional space. In a geometrical $(d+1)$-lateration method, a node can be localized by the geometrical localization when $(d+1)$ number of its neighbors have been localized and the geometrical distances between the node and those neighbors are available. References [5] and [6] prove that all the centralized and distributed implementations of the geometrical multilateration method are equivalent in localizing a $(d+1)$-lateration graph, given the graph and the anchors. Our time-bounded localization research focuses on the multilateration localization method because it can produce solutions in controllable polynomial time.

Most existing works propose physical localization schemes because they employ physical anchors. A range-free positioning method is presented in [28] based on Area-based Point-In-Triangulation test (APIT) and some super anchors that have a much larger transmission range than normal sensor nodes. Without super anchors, a bilateration-based localization method is proposed in [29] to localize sparse networks. The complexity of this proposed method is exponential. In [30], the authors propose a trilateration-based localization algorithm under the noisy distance measurement. Different triangulation-based localization schemes are proposed in [8], [9], and [31], which employ the 
relative distances toward the anchors obtained from time-difference-of-arrivals. A similar idea is employed in [32] to propose a silent self-positioning algorithm for underwater sensor networks. Another underwater localization algorithm, named 3DUL, is proposed in [33]. 3DUL employs three surface buoys as anchors and determines the internode distances through leveraging the low speed of sound. Mobile localization problem has been addressed in [34], where the actors (types of node that collect and process sensor data and perform appropriate actions) limit their location update scopes based on the Voronoi diagram, and the sensors predict the movement of the actors based on the Kalman filtering of the previously received location updates. The time bound for localization has not been addressed in the literature. The existing anchor-based works usually focus on the density of the anchors. They cannot answer determinate questions such as how many anchors are necessary and where to deploy the anchors such that the network can be localized.

Without utilizing anchors, [35] proposes a self-positioning algorithm (SPA) to build a relative coordinate system with the time-of-arrival-based range information. Local information is collected, and each node builds its local coordinate system where the node itself is located at $(0,0)$. Then, a global coordinate system is constructed by merging the local coordinate systems at the second step. The major difference between our proposed algorithm and SPA is the process of the local coordinate system construction and the time-bounded global localization. Reference [36] proposes a multidimensional scaling (MDS) localization algorithm. MDS is centralized and requires an initial estimation of the complete distance matrix. For relative localization, we propose a new concept of localizability for time-bounded deterministic study in this paper. Compared to existing works such as [35] and [36], our work is novel in that it introduces the time bound into the process of constructing local coordinate systems. We first formally define the time-bounded essential localization problem, then analyze its complexity, and finally present a practical solution.

So far, neither the theoretical nor the practical works has ever addressed the problem of time-bounded localization. Moreover, how to deploy minimum number of anchors such that the network's localizabiliy is guaranteed is still open.

\section{CONCLUSION AND FUtURE RESEARCH}

In this paper, we have explained why the traditional definition of relative localization is inappropriate for evaluating the actual efficiency of localization in practice. To address this problem, we define essential localization, a novel problem to capture the overhead of localization in a sensor network. The complexity of the problem is studied, and an efficient distributed algorithm is proposed to perform time-bounded essential localization, whose performance is evaluated through simulation studies.

The concept of essential localization is proposed to compute the minimum required time for localization and the earliest time to start a location-based application. The results presented in this paper are based on flooding-based routing protocols. They should vary when applications employ other protocols for routing. As the flooding property maximizes the information diffusion, the essential localization time derived in this paper should serve as the lower bound. In the future, our re- search will focus on investigating the essential localization time for other widely recognized routing protocols (such as shortest path and the real-time protocol SPEED [37]). In addition, the study on essential localization has a broad impact on social network applications. Therefore, we will apply the methodologies proposed in this research to the information diffusion problem in social networks.

\section{REFERENCES}

[1] M. Ding, D. Chen, K. Xing, and X. Cheng, "Location-centric storage for on-demand warning in sensor networks," in Proc. IEEE INFOCOM, Mar. 13-17, 2005, pp. 902-913.

[2] F. Liu, X. Cheng, and D. Chen, "Insider attacker detection in wireless sensor networks," in Proc. IEEE INFOCOM, Anchorage, AK, May 2007, pp. 1937-1945.

[3] K. Xing, F. Liu, X. Cheng, and D. H.-C. Du, "Realtime detection of clone attacks in wireless sensor networks," in Proc. 28th ICDCS, Beijing, China, Jun. 2008, pp. 3-10.

[4] M. Ding and X. Cheng, "Fault-tolerant target tracking in sensor networks," in Proc. 10th ACM MobiHoc, New Orleans, LA, May 18-21, 2009, pp. 125-134.

[5] W. Cheng, A. Teymorian, L. Ma, X. Cheng, X. Lu, and Z. Lu, "Underwater localization in sparse 3D acoustic sensor networks," in Proc. IEEE INFOCOM, 2008, pp. 236-240.

[6] A. Y. Teymorian, W. Cheng, L. Ma, X. Cheng, X. Lu, and Z. Lu, "3D underwater sensor network localization," IEEE Trans. Mobile Comput., vol. 8, no. 12, pp. 1610-1621, Dec. 2009.

[7] D. K. Goldenberg, P. Bihler, M. Cao, J. Fang, B. D. O. Anderson, A. S. Morse, and Y. R. Yang, "Localization in sparse networks using sweeps," in Proc. MobiCom, 2006, pp. 110-121.

[8] X. Cheng, A. Thaeler, G. Xue, and D. Chen, "TPS: A time-based positioning scheme for outdoor wireless sensor networks," in Proc. IEEE INFOCOM, 2004, pp. 2685-2696.

[9] A. Thaeler, M. Ding, and X. Cheng, "iTPS: An improved location discovery scheme for sensor networks with long range beacons," J. Parallel Distrib. Comput., vol. 65, no. 2, pp. 98-106, 2005.

[10] D. Goldenberg, "Fine-grained localization in sensor and ad-hoc networks," Ph.D. dissertation, Yale University, New Haven, CT, 2006.

[11] R. M. Karp, R. E. Miller, and J. W. Thatcher, "Reducibility among combinatorial problems," J. Symb. Logic, vol. 40, no. 4, pp. 618-619, Dec. 1975.

[12] M. Garey and D. Johnson, Computers and Intractability: A Guide to the Theory of NP-Completeness. New York: Freeman, 1979.

[13] C. C. B. N. Clark and D. Johnson, "Unit disk graphs," Discrete Math., vol. 86, pp. 165-177, 1990.

[14] P.-J. Wan, K. M. Alzoubi, and O. Frieder, "Distributed construction of connected dominating set in wireless ad hoc networks," Mobile Netw. Appl., vol. 9, no. 2, pp. 141-149, 2004.

[15] X. Cheng, X. Huang, D. Li, W. Wu, and D.-Z. Du, “A polynomial-time approximation scheme for the minimum-connected dominating set in ad hoc wireless networks," Networks, vol. 42, no. 4, pp. 202-208, 2003.

[16] X. Cheng, M. Ding, D. H. Du, and X. Jia, "Virtual backbone construction in multihop ad hoc wireless networks," Wireless Commun. Mobile Comput., vol. 6, pp. 183-190, 2006.

[17] K. Sakai, S. C.-H. Huang, W.-S. Ku, M.-T. Sun, and X. Cheng, "Timerbased CDS construction in wireless ad hoc networks," IEEE Trans. Mobile Comput., vol. 10, no. 10, pp. 1388-1402, Oct. 2011.

[18] J. Blum, M. Ding, A. Thaeler, and X. Cheng, "Connected dominating set in sensor networks and MANETs," in Handbook of Combinatorial Optimization. New York: Springer, 2005, pp. 329-369.

[19] S. Kutten and D. Peleg, "Fast distributed construction of small k-dominating sets and applications," J. Algor., vol. 28, no. 1, pp. 40-66, 1998.

[20] L. D. Penso and V. C. Barbosa, "A distributed algorithm to find k-dominating sets," Discrete Appl. Math., vol. 141, no. 1-3, pp. 243-253, 2004.

[21] F. Zou, Z. Zhang, and W. Wu, "Latency-bounded minimum influential node selection in social networks," in Proc. 4th WASA, 2009, pp. $519-526$.

[22] X. Zhu, J. Yu, W. Lee, and D. Kim, "New dominating sets in social networks," J. Global Optimiz., vol. 48, no. 4, pp. 633-642, 2010.

[23] J. Aspnes, T. Eren, D. K. Goldenberg, A. S. Morse, W. Whiteley, Y. R. Yang, B. D. O. Anderson, and P. N. Belhumeur, "A theory of network localization," IEEE Tran. Mobile Comput., vol. 5, no. 12, pp. 1663-1678, Dec. 2006. 
[24] J. Saxe, "Embeddability of weighted graphs in k-space is strongly np-hard," in Proc. 17th Allerton Conf. Commun., Control, Comput., 1979 , pp. $480-489$.

[25] R. Connelly, "Generic global rigidity," Discrete Comput. Geom., vol. 33 , no. 4 , pp. 549-563, 2005.

[26] B. Hendrickson, "Conditions for unique graph realizations," SIAM J. Comput., vol. 21, no. 1, pp. 65-84, 1992.

[27] B. Jackson and T. Jordán, "Connected rigidity matroids and unique realizations of graphs," J. Combin. Theory Ser. B, vol. 94, no. 1, pp. $1-29,2005$.

[28] T. He, C. Huang, B. M. Blum, J. A. Stankovic, and T. Abdelzaher, "Range-free localization schemes for large scale sensor networks," in Proc. 9th Annu. MobiCom, 2003, pp. 81-95.

[29] D. K. Goldenberg, P. Bihler, M. Cao, J. Fang, B. D. O. Anderson, A. S. Morse, and Y. R. Yang, "Localization in sparse networks using sweeps," in Proc. 12th Annu. MobiCom, 2006, pp. 110-121.

[30] D. Moore, J. Leonard, D. Rus, and S. Teller, "Robust distributed network localization with noisy range measurements," in Proc. 2nd SenSys, 2004, pp. 50-61.

[31] F. Liu, X. Cheng, D. Hua, and D. Chen, "Location discovery for sensor networks with short range beacons," Int. J. Ad Hoc Ubiq. Comput., vol. 4, no. 3/4, pp. 125-136, Apr. 2009.

[32] X. Cheng, H. Shu, Q. Liang, and D. H.-C. Du, "Silent positioning in underwater acoustic sensor networks," IEEE Trans. Veh. Technol., vol. 57, no. 3, pp. 1756-1766, May 2008.

[33] M. T. Isik and O. B. Akan, "A three dimensional localization algorithm for underwater acoustic sensor networks," IEEE Trans. Wireless Commun., vol. 8, no. 9, pp. 4457-4463, Sep. 2009.

[34] T. Melodia, D. Pompili, and I. F. Akyldiz, "Handling mobility in wireless sensor and actor networks," IEEE Trans. Mobile Comput., vol. 9 , no. 2, pp. $160-173$, Feb. 2010.

[35] S. Capkun, M. Hamdi, and J. Hubaux, "Gps-free positioning in mobile ad-hoc networks," in Proc. 34th Annu. HICSS, 2001, vol. 9, p. 9008.

[36] Y. Shang and W. Ruml, "Improved MDS-based localization," in Proc. IEEE INFOCOM, 2004, pp. 2640-2651.

[37] T. He, J. A. Stankovic, C. Lu, and T. Abdelzaher, "SPEED: A stateless protocol for real-time communication in sensor networks," in Proc. 23rd ICDCS, 2003, pp. 46-55.

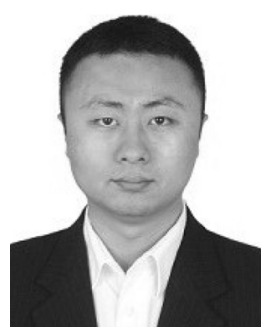

Wei Cheng (M'11) received his Ph.D. degree in computer science from The George Washington University, Washington, DC, in 2010.

He is a Research Assistant Professor with the Department of Computer Science, University of Massachusetts Lowell, Lowell. His research interests include localization, RFID system on road, cyber physical systems, security, wireless networks, and polynomial-time algorithm design and analysis.

Dr. Cheng is a member of the Association for Computing Machinery (ACM).

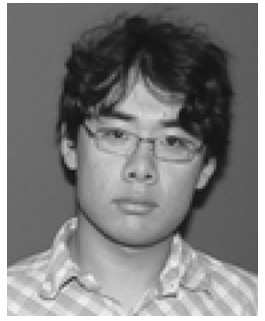

Nan Zhang (M'06) received the B.S. degree from Peking University, Beijing, China, in 2001, and the $\mathrm{Ph} . \mathrm{D}$. degree from Texas A\&M University, College Station, in 2006, both in computer science.

$\mathrm{He}$ is an Assistant Professor with the Department of Computer Science, The George Washington University, Washington, DC. His research interests focus on databases, information security and privacy, and distributed systems.

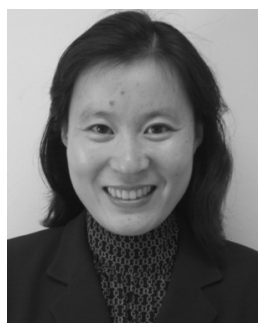

Xiuzhen Cheng (M'03-SM'07) received the M.S and Ph.D. degrees in computer science from the University of Minnesota, Twin Cities, Minneapolis, in 2000 and 2002, respectively.

She is an Associate Professor with the Department of Computer Science, The George Washington University, Washington, DC. She worked as a Program Director for the US National Science Foundation (NSF) for six months in 2006 and joined the NSF again as a part-time Program Director in April 2008 Her current research interests include cyber physical systems, wireless and mobile computing, sensor networking, wireless and mobile security, and algorithm design and analysis.

Dr. Cheng received the NSF CAREER Award in 2004

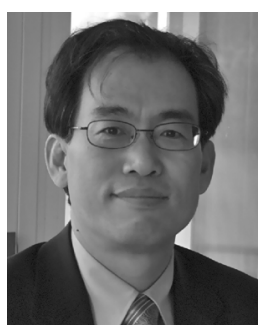

Min Song (M'01-SM'07) received the Ph.D. degree in computer science from the University of Toledo, Toledo, $\mathrm{OH}$, in 2001

$\mathrm{He}$ is currently a Program Director with the Division of Computer and Network Systems, Directorate for Computer and Information Science and Engineering, National Science Foundation, Washington, DC. He is also a Professor with the Electrical Engineering and Computer Science Department, University of Toledo. His research interests include design, analysis, and evaluation of cognitive radio networks, wireless sensor networks, wireless mesh networks, WLAN, mobile ad hoc networks, and network security.

Dr. Song is the recipient of the NSF CAREER Award and the DOE GAANN Award.

Dechang Chen received the Ph.D. degree in statistical learning from the State University of New York at Buffalo in 1998.

Currently, he is an Associate Professor with the Division of Epidemiology and Biostatistics, Uniformed Services University of the Health Sciences, Bethesda, MD. His main research interests include statistical learning and its applications (in bioinformatics, computational medicine, and ad hoc sensor networks). $\mathrm{He}$ also conducts research in applied statistics and partial differential equations. 